(Aus dem physiologischen Institut der Universität Rom.)

\title{
Über eine besondere Druckempfindlichkeit der Glans penis.
}

\author{
Ein Beitrag zur Kenntnis der an dem Geschlechtsakte \\ teilnehmenden peripheren Empfindungen. \\ Untersuch ungen \\ von
}

S. Haglioni.

Beim heutigen Stand unserer Kenntuisse gilt die Annahme, dass die physiologische Analyse nicht vernag, in der Haut der äusseren Geschlechtsorgane (Begattungsorgane) irgendeine spezifische Form Elementarempfindungen nachzuweisen, die dieselben vor den übrigen Hautgegenden auszeichnen. Die nachweisbaren Unterschiede bestehen nur in der Abwesenheit oder Spärlichkeit einiger allgemeinen Hautsinne (Tast- und Wärmesinn). Diese Meinung fusst sich hauptsächlich auf die Versuchsergebnisse von v. Frey ${ }^{1}$ ), der durch punktförmige Reizung mit abstufbaren mechanischen Reizen (Reizhaare) die Tast- und Schmerzempfindlichkeit, mit erwärmten oder abgekühlten Metalldrähten die Temperaturempfindlichkeit der Haut des Penis, von dessen Wurzel bis zum Präputium, zu der Eichel, zu der Urethralmündung untersuchte. Er fand, dass die an der Wurzel des Gliedes spärlich vorhandenen Druckpunkte dichter stehen, je mehr man sich dem Rande der Vorhaut nähert; auf dem inneren Blatte der Vorhaut, gegen den Eichelhals. nehmen sie ab und verschwinden schliesslich. Das Frenulum ist reich an Druckpunkten, welche in der ganzen Haut der Eichel fehlen. Mechanische Reizungen

1) M. v. Frey, Beiträge zur Sinnesphysiologie der Haut. III. Mitt: Ber. d. kgl. sächs. Gesellsch. d. Wissensch. zu Leipzig, math.-physik. Klasse Bd. 47 S. 166-184. 1894. Die Ergebnisse findet man auch im vierten Band der Luciani'schen Physiologie des Menschen ausführlich wiedergegeben. 
der Eichel, welche das Glied als Ganzes bewegen, werden durch den Drucksinn der genannten Orte in Form der gewöhnlichen Druckempfindungen wahrgenommen. Fasst man dagegen mit einer Pinzette eine Falte der Eichel und vermeidet eine Bewegung des Gliedes, so wird bei schwachem Kneifen nichts gefühlt, bei starkem Kneifen Schmerz. Das Präputium reagiert auf denselben Eingrifi zuerst mit Druckempfindung, weiterbin mit Schmerz, entsprechend der doppelten Reizschwelle. Übereinstimmende Ergebnisse liefert die faradische Reizung. Der Temperatursinn, namentlich in Form von Kaltpunkten, ist dagegen in der Haut des Eichelhalses und der Corona glandis besonders ausgebildet.

"Die Ansicht [schliesst Luciani ${ }^{1}$ ) mit v. Frey] derer, welche in der Aussenfläche der Genitalorgane einen spezifischen Sinn für die Wollustempfindungen annehmen, entbehrt also jeder Begründung. Die Reizung der Tastpunkte des Penis (und vielleicht auch der Schmerz- und Temperaturpunkte der Glans) kann sicher mit Wollustempfindungen verknüpft sein, wie dies auch bei anderen besonderen Regionen der Haut und bei anderen Schleimhäuten der Fall ist; aber diese Verknüpfung ist weder notwendig noch konstant und hängt sehr wahrscheinlich von dem Zusammentreffen besonderer Erregbarkeitsbedingungen der Sinneszentren ab."

Gelegentlich einiger Untersuchungen über die Wirkung des faradischen Stromes auf die Glans ${ }^{2}$ ) bemerkte ich auf der Corona glandis von S. B. eine Anzahl deutlich sichtbarer, der Längsachse des Penis radialwärts geordneter, mit einer besonderen Empfindlichkeit begabten Hautpapillen. Wenige Verfasser ${ }^{3}$ ) erwähnen diese

1) A. a. O. S. 82 .

2) S. Baglioni, Contributo analitico alla cosidetta sensibilitá elettrica Riv. di psicologia applicata vol. 6, 1910.

3) Henle schreibt S. 436 des zweiten Bandes seines Handbuches (2. Aufl., Braunschweig 1873): „Auf der gewölbten Fläche der Glans stehen die zahlreichen Papillen in Längsreihen, die gegen das Orificium urethrae konvergieren; in derselben Richtung nehmen die Papillen an Stärke zu, doch sind sie alle in der Dicke der Epidermis versteckt. Grössere Papillen, die als weisse Flecke von 0,5-1 mm Durchmesser durch die Epidermis schimmern oder sie hügelartig erheben, stehen öfters auf der Corona glandis, dicht gedrängt oder zerstreut, in einfacher oder mehrfacher Reihe, um so grösser, je spärlicher sie sind." In einer Fussnote erwähnt Henle ferner, dass diese in der Corona sichtbaren Papillen von älteren Anatomen für die umstrittenen, angeblich Smegma praeputii 
anatomische Besonderheit, da sie nicht häufig ${ }^{1}$ ) vorkommt. Denn bei den meisten Menschen hat die Corona das gleiche Aussehen wie die übrige Glans, indem ihre Aussenfläche glatt und frei von aussenher wahrnehmbaren Erhebungen ist. Die nämliche Besonderheit (mit einigen kleinen Nebenunterschieden) konnte ich jedoch in der Corona von G.P. feststellen, an welchem ich die an S. B. erzielten Ergebnisse kontrollieren konnte. Zur Ausschliessung jeden Zweifels, dass die fraglichen Bildungen pathologischer Herkunft (etwa kleine Kondylome) seien, füge ich hinzu, dass beide Versuchspersonen an keiner örtlichen Erkrankung litten. Übrigens werde ich zeigen, dass auch die Individuen mit glatter Corona eine besondere Empfindlichkeit in dieser Gegend besitzen.

\section{Aussehen der mit Papillen besetzten Corona.}

a) Die Corona glandis von S. B. ist von kleinen Erhebungen (Papillen) besät, die in den verschiedenen Stellen an Grösse und Zahl schwanken. Die übrigen. Teile der Eichel, deren Hals sowie das Präputium weisen eine glatte, glänzende Oberfläche auf. Die einzelnen Papillen der Corona sind kegelähnlich, mit einer breiten abgeplatteten Basis; die grössten haben unter gewöhnlichen Zuständen des Gliedes eine Höhe von $1 \mathrm{~mm}$ und eine Basisbreite von $1 \mathrm{~mm}$, die übrigen haben kleinere Dimensionen; die geringsten erscheinen

sezernierenden Tyson'schen oder Littre'schen Drüsen gehalten wurden. G. Simon (Über die sogenannten Tyson'schen Drüsen an der Eichel des männlichen Gliedes, Müller's Arch. 1844 S. 1-8), ein Schüler Valentin's, hatte indessen durch histologische Untersuchnngen nachgewiesen, dass es sich um Hautpapillen handelt, in denen Empfindungsnerven enden. „Aus dem Gesagten (so schloss daraus Simon) ergibt sich, wie ich glaube, mit Bestimmtheit, dass die sogenannten Tyson'schen Drüsen kleine Erhebungen der Cutis sind, deren Oberfläche mit Gefühlswärzchen besetzt ist. Das Smegma praeputii kann mithin auch durch sie nicht gebildet werden, sondern sie haben wahrscheinlich den Zweck, die Empfindlichkeit der Eichel zu vermehren." Diese Hypothese findet in den vorliegenden Untersuchungen tatsächlich ihren experimentellen Beweis.

Auch C. J. Eberth (Die männlichen Geschlechtsorgane, Bardeleben's Handb. S. 206. Jena 1904) erwähnt die Papillen der Eichel, ohne sie jedoch in der Corona zu lokalisieren. „Bei vielen Individuen ist die Oberfläche (der Glans) durch spärliche, 0,1-0,5 mm hohe, an Zahl schwankenden Höckerchen oder Papillen rauh."

1) Nach H. Sprünck (Über die vermeintlichen Ty son'schen Drüsen, Inaug.-Dissert. Anatom. Institut Königsberg, zitiert aus Eberth) hatten unter 300 Individuen 183 eine glatte Eichel $(61 \%)$. 
als rundliche, kaum aus der Fläche herausragende Punkte. Ihre Konsistenz ist derjenigen der übrigen Eichelbaut ähnlich. Sie sind in radialwärts verlaufenden Reihen eingeordnet, von denen aber nur die erste vordere Reihe, am oberen Rand der Corona gelegen, und die letzte hintere Reibe, an der Grenze vor dem Eicluelhals, regelmässig in einem Abstande von 1-2 mm voneinander geordnet sind. Die in der zwischenliegenden Region der Corona vorkommenden Papillen liegen zerstreut. Bezüglich ihrer Dimensionen bilden im allgemeinen die grössten die letzte hintere Reihe der Coronabasis, die mittelmässigen befiuden sich in der mittleren Gegend, die kleinsten in der ersten vorderen Reihe. In dem Maasse, in dem man von der medianischen Gegend des Coronarückens aus seitwärts bis zum Frenulum fortschreitet, nehmen die Papillen an Zahl und Grösse ab, bis sie schliesslich ausbleiben; das Drittel der Corona in ter Umgebung des Frenulums wird somit papillenfrei. Ein anderes Merkmal der Papillen liegt in ihrer Richtung; diejenigen nämlich, welche in der rechten Hälfte der Corona sitzen, sind mit ibren Spitzen nach rechts gerichtet, diejenigen der linken Hälfte nach links. Bei der Erektion nehmen sie um das Zweifache und mehr zu, sich deutlich von der Grundfläche erbebend. Ihre Gesamtzabl beträgt etwa 100 . Sie sind sämtlich einfach.

b) Bei G.P. befinden sich die Papillen in den seitlichen Gegenden der Corona; in den mittleren Gegenden des Rückens und des Frenulums sind nur schwache Erhebungen der Haut vorhanden, denen jedoch der morphologische Wert wenig ausgebildeter Papillen beizumessen ist. Links können 24 starke und fast sämtlich in einer einzigen Reihe geordnete Papillen leicht gezählt werden; rechts können deren 40 gezählt werden, die zum grossen Teil in einer lıiuteren Reihe (an der Basis der Corona), zum kleinen Teil in einer zweiten mittleren Reihe und nur einige wenige in eirer dritten vorderen Reihe vorkommen. Bezüglich ihrer Gestalt können sie eingeteilt werden in einfache (kleine Kegel mit abgestumpfter Spitze oder kleine Zylinder mit rundlichem Ende oder kleine niedrige Kolben) und zusammengesetzte, welche sich aus der Verschmelzung zweier oder mehrerer einfacher Papillen zu ergeben scheinen. Ihre Länge (oder Höhe) schwankt zwischen 0,5 bis etwa $2 \mathrm{~mm}$ beim schlaffen Gliede; beim Erektionszustande und bei passiv herbeigeführter Kongestion nehmen sie an Grösse zu, während die kleinen Hauterhebungen, die, wie gesagt, in den übrigen Teilen der Corona be- 
obachtet werden, deutlicher dabei werden. Die Richtung der Papillen läuft hier von hinten nach vorn, d. b. nach der Harnröhrenmündusg zu.

Weitere anatomische Untersuchungen werden von A. Ruffini ausgeführt, über die er an anderem Orte berichten wird.

\section{Physiologische Uutersuchungen.}

Die physiologischen Untersuchungen bestanden in der Feststellung der Eigenschaften und der Versuchsbedingungen der Empfindlichkeit der genannten Papillen und der angrenzenden Hautgegenden der Eichel. Iusbesondere wurde die Wirkung der mechanischen (Druck-) Reize untersucht; dadurch wurden folgende Hauptergebnisse erzielt.

Die angewendeten mechanischen Reize waren verschiedener Art:

a) punktförmige Reize von abstufbarer Stärke (Reizhaare von v. Frey, Glashaare nach Alrutz, Nadeln);

b) breitflächige Reize verschiedener Stärke (Haarpinsel, Wattebäuschchen, abgerundetes Ende eines Glasstabes, Pinzette, Fingerbeere).

Beiläufig wurden auch faradische und thermische Reize geprüft, mit denselben Ergebnissen wie v. Frey.

Die Wirknng der nechanischen Reize ist wesentlich verschieden, je nach der Art der Reize:

a) Die auf der Aussenfläche der ganzen Eichel (einschliesslich der Corona) applizierten punktförmigen Reize erzeugen entweder Schmerz oder keinerlei Empfindung. Darin haben wir eine völlige Bestätigung der Resultate v. Frey's, der daraus folgerte, dass die Glans frei von I)ruckpunkten sei.

b) Anders ist die Wirkung von Druckreizen, welche mehrere Hautpunkte zu gleicher Zeit oder rasch aufein ander treffen. Wird zunächst mit dem abgerundeten Ende des Glasstabes oder einem äbnlichen Gegenstand die Eichel gedruckt, so wird eine dunkle Druckempfindung wahrgenommen, die man nicht imstande ist, genau zu lokalisieren und deren Eigenschaften auseinanderzusetzen. Man könnte z. B. nicht angeben, ob die Reizfläche glatt oder rauh ist. Doch ist es eine schmerzfreie Empfindung. Eine ähnliche, jedoch bedeutend stärkere Empfindung wird von gleichen Druckreizen hervorgerufen, die mehrere Punkte der Eichelfläche $\mathrm{rasch}$ nache in a d e r und zu wi ed e rhol ten Mal en treffen. Dies verwirklicht 
sich, wenn man die Haut mit dem Reizgegenstand streift. Als der geeignetste, d. h. die grösste Folge herbeiführende Reiz stellte sich dabei die mit Vaseline schlüpfrig gemachte Zeigefingerbeere heraus; und die Eichelgegend, welche für diese Reizart am empfindlichsten sich erwies, war eben die mit Papillen versehene Region der Corona. Die dadurch auftretende Empfindung trägt in kurzen Worten die wesentlichen Eigenschaften der tiefgehenden, in mancher Hinsicht dunklen (weil schwer in ihren Elementen analysierbar) Geschlechtswollust. Durch solche, auf die Corona glandis beschränkte Reizungen wird leicht der Erektionsreflex ausgelöst. Zweifellos ist hauptsächlich diese Gegend der Entstehungsort der Erregungen, die beim Koitus (resp. der Masturbation), eben durch ähnliche, rhythmisch aufeinanderfolgende mechanische Reize der ganzen Corona hervorgerufen, den Ejakulationsreflex auslösen und zu dem Wollustgefühle wesentlich beitragen.

Des näheren fand ich, dass der grösste Effekt dadurch erzielt wirđ̄, wenn die sukzessive Reizung der Papillen in entgegengesetztem Sinue ihrer Richtung erfolgt. Bei S. B. z. B., dessen Papillen seitwärts gerichtet sind, erreicht die Empfindung den grössten Grad, wenn die Papillen von aussen medialwärts gestreift, d. h. passiv erhoben werden. Jeder wie auch geartete Druckreiz, örtlich auf die Papillen angebracht, mittlerer Stärke, weckt in denselben die erwähnte Lustempfindung. Wird aber diese Empfindung bloss in einer oder wenigen Papillen erzeugt, so hat die dabei auftretende Empfindung einen unvergleichlich geringeren Grad als die durch Reizung einer grösseren Anzahl Papillen entstehende Empfindung.

Äusserst schwer, ja geradezu unmöglich ist die Analysierung der einzelnen Eigenschaften der durch die erwähnten adäquaten Reize hervorgerufenen Lustempfindung. Man kann nur behaupten, dass sie (abgesehen von ihrer wesentlichen Ähnlichkeit mit der Geseblechtswollust, von der sie sich in dem Stärkegrad quantitativ und nicht qualitativ unterscheidet) mitunter, namentlich sofort nach der Ejakulation, eine leichte Schmerztönung erweist.

Hinzugefügt sei ferner, dass diese besondere Empfindlichkeit, selbstredend bei Anwendung derselben Reize, auch in den übrigen Gegenden der Eichelhaut festzustellen ist, wo sie aber einen durchaus geringeren Stärkegrad bat. Auf dem Frenulum ist sie vielleicht zum Teil noch vorhanden, wo sie aber einen besonderen Charakter 
dadurch annimmt, dass sie sich mit der hier vorbandenen hohen Tastempfindlichkeit vermischt. Sie fehlt jedoch in der Haut des Präputiums und des Penis, wo die genannten Reize mit den bekannten Eigenschaften des Tastsinnes klar empfunden werden. Man erhält die qualitativ verschiedenen lokalen Empfindungsarten unter Anwendung einer und derselben Reizart sehr leicht nacheinander, wenn man mit der Fingerbeere von der Harnröhrenmündung aus die versehiedenen Hautgegenden des Eichelrückens, der Corona, des Eichelhalses, des zurückgehaltenen Präputiums der medianen, dorsalen Linie entlang nacheinander streift. Zuerst wird dann eine vage dumpfe wollüstige Druckempfindung wahrgenommen, die namentlich in der Tiefe sich ausbreitet und ihren grössten Stärkegrad auf der Corona erreicht, um sich darauf beim Übergang auf das Präputium im Eichelhalse plötzlich in eine wahre deutliche und gut lokalisierbare Tastempfindung zu verwandeln.

Die besondere Empfindlichkeit der Corona und der Eichel ist beträchtlichen Schwankungen in ihrer Stärke unterworfen, je nach den Zuständen der Nervenzentren und der übrigen Geschlechtsorgane. Sie erreicht den höchsten Grad nach einer Periode geschlechtlicher Abstinenz, wird minimal (und nimmt einen deutlichen schmerzlichen Charakter) sofort nach dem Koitus.

Ein derartiges Verbalten ist jedoch nicht der mit Papillen versehenen Eichel eigentümlich. Ähnliche an G. A., der eine vollkommen glatte Eichel besitzt, angestellten Untersuchungen ergaben gleiche Resultate. Denn auch er empfand durch Anwendung der genannten adäquaten Druckreizung die besondere Lustempfindung, die ebenfalls ihren höehsten Stärkegrad auf der Corona erreichte. Demnach wären die sichtbaren Papillen nur als sekundäre Bildungen zu deuten, die nur bei einigen Individuen zutage treten, vielleicht als Organe, die die besondere Empfindlichkeit zu erhöhen befähigt sind. Mit anderen Worten besitzen wahrscheinlich alle gesunden geschlechtsreifen Männer grosse sensible, in Aer Dicke der Eichelhaut versteckte Papillen (Henle, s. oben S. 362); nur bei einigen Individuen erheben sie sich in der Corona oberhalb der Aussenfläche als sichtbare Höckerchen. Es ist jedoch die Frage nicht leicht $z \mathfrak{u}$ beantworten, ob letztere Individuen tatsächlich eine höhere Lustempfindung erfahren. Weniger schwer und vom wissenschaftlichen Standpunkte aus etwa wichtiger - scheint die 
Lösung der Frage, in welchem Zeitalter die genannten Papillen auftreten und bzw. verschwinden ${ }^{1}$ ), ebenso wie die Frage nach dem Vorhandensein der besprochenen besonderen Druckempfindung bei den Alten und den Impotenten.

Von dem Gedanken geleitet, dass die Aussenfläche der Eichel (namentlich der Corona) der Sitz der besonderen Druckempfindungen ist, die beim Koitus den Ejakulationsreflex zum grossen Teile auslösen, nahm ich mir vor, die Folgen der mittels lokaler Applikation einer $5 \%$ igen Stovainlösung herbeigeführten vorübergehenden Anästhesie der Eichelhaut beim Koitus festzustellen. Es erfolgte eine spärliche Ejakulation mit beträchtlicher Verspätung, d. h. nach 14 Minuten anstatt 3 Minuten (wie die gewöhnliche, unter sonstigen gleichen Bedingungen statthabende Koitusdauer ist). Ferner erreichte beim Akte die Wollust nie den gewohnten Höhepunkt; man spürte nur eine unvollständige Fmpfindung, als ob der Penis von einer dicken Kondommembran bekleidet wäre.

Die Ergebnisse dieses Versuches bestätigen also die Aunahme, dass die Haut der Glans (insbesondere der Corona) tatsächlich der Entstehungsort der afferenten Erregungen ist, die zu der Auslösung des Ejakulationsreflexes und zu dem gesamten Wollustgefühle wesentlich beitragen. Sie lassen ferner den praktischen Gebrauch einer derartigen vorübergehenden Anästhesie (bzw. Hypoästhesie) der Eichelhaut bei Fällen voreiliger Ejakulation für berechtigt erscheinen.

Andererseits beweisen sie aber, dass es vielleicht ein Irrtum wäre, anzunehmen, dass der Ejakulationsreflex und vor allem das komplexe Wollustgefühl ausschliesslich von den mechanischen Reizungen der Eichel abhängen. Bei Beschränkung auf die Betrachtung anderweitiger peripherer Erregungen, namentlich abgesehen von den jeweiligen zentralen oder psychischen Zuständen, deren weitgehender Einfluss auf die in Rede stehenden Vorgänge allgemein anerkannt ist, nehmen zu deren Zustandekommen zweifellos andere Empfindungen teil, wie z. B. die Tastempfindungen der Penishaut und der Haut anderer Gegenden, die inneren Druckempfindungen der Ausführungs-

1) Durch die Untersuchungen Nussbaum's ist heute bekannt, dasd bei den Frosch- (und Kröten-) Männchen die empfindlichen Papillen ihrer Daumen erst bei der Brunstzeit sich entwickeln. Bei anderen Säugetieren, z. B. beim Meerschweinchen, besitzt die Eichel ebenfalls zahlreiche Papillen, denen vielleicht dieselbe physiologische Aufgabe obliegt. 
gänge des Samens, der an der Ejakulation sich beteiligendeu Muskeln usw.

Die Haut der Eichel, insbesondere der Corona (mit oder ohne Papillen), wäre jedoch sozusagen die am meisten von peripheren Aufnahmeorganen, die auf besondere adäquate mechanische Reize ansprechen, reich besetzte reflexauslösende Zone. Infolgedessen kann der von v. Frey aufgestellte Satz, dass in der Aussenfläche der Glans keine weitere Empfindungsformen, ausser des Schmerz- und Temperatursinnes, nachweisbar sind, nicht mehr absoluterweise aufrechterhalten werden. Offenbar sind seine verneinenden Ergebnisse auf die Versuchsınethodik zurückzuführen ; denn er hat keine adäquaten mechanischen Reize angewendet. Zum Schlusse sei hinzugefügt, dass tatsächlich selbst die faradische Reizung der Corona (und deren Papillen) bezuglich der besonderen Lustempfindung sich unwirksam erwies.

Unsere Ergebnisse zusammenfassend werden wir also schliessen:

1. In der Haut der Eichel wird unter Anwendung adäquater mechanischer Reize, die in zu gleicher Zeit oder rasch aufeinanderfolgenden Druckwirkungen auf breitfächige Hautzonen bestehen, eine besondere Empfindungsart nacbgewiesen, die ibren höchsten Stärkegrad auf der Corona und bei wiederholter Reibung mit eingefetteter Fingerbeere erreicht.

2. Bei einigen Individuen ist die Coronahaut bei einigen Stellen von deutlichen $(0,5$ bis $1-2 \mathrm{~mm}$ beim Schlaffzustande, $2-3 \mathrm{~mm}$ bei Erektion) einfachen oder zusammengesetzten Papillen besetzt. Es sind dann diese Papillen der Sitz der grössten Empfindung, welche durch jede, ihre Spitzen erhebende passive Verlagerung erweckt wird. Auch die Individuen, welche eine glatte Corona haben, erfabren jedoch unter den gleichen Bedingungen dieselbe Empfindung.

3. Die dadurch hervorgerufene Empfindung unterseheidet sich von den gewöbnlichen Tastempfindungen (die bekannterweise in der Eichelhaut fehlen) auch deswegen, weil sie einen bedeutend höheren Schwellenwert hat, ist subtil, lustbetont, erinnert deutlich an die sexuelle Wollust. In ihren Wahrnehmungsmerkmalen ist sie jedoch dunkel, schwer lokalisierbar, weil sie sich besonders in die Tiefe ausbreitet, kann auch mitunter, namentlich nach der Ejakulation, eine schwache Schmerzfärbung aufweisen.

4. Der Erregbarkeitsgrad wechselt sehr nach den jeweilig herrschenden Zuständen der Zentren und der übrigen Geschlechts- 
370 S. Baglioni: Über eine besondere Druckempfindlichkeit der Glans penis.

organe; die höchste Stärke tritt bei Anbäufung des Hodensekrets auf; sofort nach der Samenentleerung wird die Erregbarkeit minimal unter Umwandlung einiger Charaktere der Empfindung, die eine leichte Schmerztönung annimmt.

5. Durch vorherige Stovainisierung herbeigefübrte örtliche Anästhesie (oder Hypoästhesie) der Eichelhaut verlängert die Dauer des Ejakulationsreflexes und stumpft das Wollustgefühl ab.

In der Haut der Eichel, namentlich der Corona, gibt es also periphere nervöse Aufnahmeorgane, die mit einer besonderen mechanischen Empfindungsform begabt sind, welche in hohem Maasse zur Auslösung der sexuellen Reflexe und zum Zustandekommen der Wollust beitragen. Auf diese Hautgegend trifft die Bezeichnung reflexogene Hautzone der Begattung zu, ebenso wie auf die in derselben vorhandenen Nervenendorgane der Name periph ere Wollustorgane. 